\title{
Data matters: implications for surgery and anesthesia in achieving universal health coverage
}

\author{
Julian Gore-Booth, MA $\cdot$ Jannicke Mellin-Olsen, MD, DPH
}

Received: 10 September 2018/ Accepted: 10 September 2018/Published online: 20 November 2018

(C) Canadian Anesthesiologists' Society 2018

Data not only measure change, but also inspire and target it. Without data it is very difficult, if not impossible, to accelerate and sustain lasting progress.

The era of the millennium development goals (MDGs) helped highlight the importance of data for development. ${ }^{1}$ Historically, most data collected globally included measures such as population, gross national product, or gross domestic product per capita, which were predominantly economic or demographic indicators. It is only later that indicators began to include more detail of the human experience. One of the most ground-breaking, and certainly influential changes, came in 1990 with the first Human Development Report ${ }^{2}$ and the human development index (HDI). The HDI (based on life expectancy, per capita income, and education) remains an important source of data and country level data comparison almost 30 years later. This is due, in large part, to the fact that it produces a composite ranking based on just a few indicators that are widely collected, comparable, and meaningful.

The MDGs were announced in September 2000 by the United Nations General Assembly. The first monitoring framework was made up of 18 targets and 48 indicators responding to eight overall goals. Twenty-three of these indicators were health-related. Goal four was "reduce child mortality" and goal five was "improve maternal health". Related indicators included "[age] under-five mortality rate" and "maternal mortality rate". These indicators are now collected by almost every country in the world. Both are key drivers and measures of change, with huge impact

J. Gore-Booth, MA $(\bowtie) \cdot$ J. Mellin-Olsen, MD, DPH World Federation of Societies of Anaesthesiologists, Dean Bradley House, 52 Horseferry Rd, London SW1P 2AF, UK e-mail: ceo@wfsahq.org on global health decision-making, and financing the sustainable development goals, ratified in 2015, have 232 indicators; over 50 of these are health-related. ${ }^{3}$ Nevertheless, not a single one of these 50 relate specifically to anesthesia and surgery, despite the fact that approximately $33 \%$ of all deaths in 2010 were from conditions amenable to surgical intervention. ${ }^{4}$ On another globally recognized indicator set, the World Health Organization's (WHO) global reference list of 100 core health indicators, ${ }^{5}$ only four relate directly to anesthesia and surgery:

- Perioperative mortality rate: all cause death rate prior to discharge among patients having one or more procedures in an operating room during the relevant admission;

- Surgical Volume: procedures performed in an operating room per 100,000 population per year;

- Access to emergency surgery: percentage of the population that can access (within two hours) a facility that can perform emergency Cesarean delivery, laparotomy, and open fracture fixation;

- Health worker density and distribution: density of health workers (i.e., surgeons, anesthesiologists, and obstetricians) per 10,000 population.

Two additional indicators (proportion of the population facing catastrophic or impoverishing health expenditure) can be disaggregated to look at surgical expenditure alone. These indicators are also included in the World Bank's World Development Indicators. ${ }^{6}$

The inclusion of these four indicators required a concerted effort and will require considerable ongoing effort for collection and analysis. It is in this context that the article from Juran et al. published in this issue of the Journal is both welcome and timely. ${ }^{7}$ As the authors point 
out, it seems that the world has finally acknowledged the connection between the goal of universal health coverage by 2030 and health system strengthening. ${ }^{8}$ Yet, there is still a disconnect between achieving the strengthened health systems and coverage we all want, and the recognition that this must include anesthesia and surgery. Cost is often cited as one reason for not doing this. Nevertheless, the estimated $\$ 350$ billion cost to scale up in low- and middle-income countries (LMICs) to meet the target of 5,000 surgical procedures per 100,000 population per year is a fraction of the $\$ 12.3$ trillion saving that could be made in these same countries by avoiding losses in economic productivity (estimated cumulative loss 2015-2030). ${ }^{4}$ It also represents just $4 \%$ of the $\$ 7.83$ trillion global spend on health in $2013 .{ }^{9}$ With total spending on health expected to increase to $\$ 18.3$ trillion by 2040 , we need better data to ensure that some of that increase goes to global anesthesia and surgery.

\section{The response}

Therefore, the major gap in knowledge with respect to surgical inequities that Juran et al. highlight is concerning. The World Federation of Societies of Anaesthesiologists (WFSA) is one organization that has been working to address this gap. Indeed, in September 2017, we have published the Global Anaesthesia Workforce Survey as the first truly global attempt to gather data about both physician and non-physician anesthesia providers. ${ }^{10}$ This goes together with an online workforce map ${ }^{11}$ that provides a more interactive and up to date visual presentation of where in the world the deficit of anesthesiologists is most acute. In this way, the survey and map become useful tools to influence change and to measure progress. The WFSA aims to update the map regularly, as well as repeat the survey every four years. Repeating the survey also ensures that we can adapt and improve. For example, we hope to collect the same workforce data, but disaggregated by sex, when we repeat the survey in 2019-20.

In addition, the WFSA has produced the Anaesthesia Facility Assessment Tool (AFAT). ${ }^{12}$ Based on the recently updated 2018 WHO-WFSA International Standards for a Safe Practice of Anesthesia, ${ }^{13}$ the WFSA has developed the AFAT to help regional and national anesthesia and healthcare leadership to gather comprehensive data about anesthesia workforce, equipment, medicines, and practice at the facility level. The AFAT is part of a shared effort to improve data collection and knowledge management in support of the implementation of World Health Assembly Resolution 68.15, which calls on all member states to strengthen emergency and essential surgical care and anesthesia in the context of universal health coverage. ${ }^{14}$
It will also help ensure that anesthesia is represented in national health planning, including national surgical, obstetric, and anesthesia plans. ${ }^{15}$

\section{Collective action for data collection}

All this depends on partnership and teamwork, not only the surgical team, but national governments, international organizations, academia, non-governmental organizations, patients, and others. The WHO, the United Nations Statistical Commission, and the World Bank are central to this effort. They will need to work together to ensure not only that anesthesia and surgical indicators are included in their datasets, but that we are collecting, sharing, and analysing the same available, comparable, and useful data across the global health system.

Juran et al. highlight the need for an international conference or seminar. In this regard, the WFSA is working closely with the Laerdal Foundation, the WHO, King's College London, former leaders of the Lancet Commission on Global Surgery, Lund University, University of Gothenburg, Harvard Medical School, and the University of California, San Francisco's global anesthesia team and others, to convene an Utstein meeting ${ }^{16}$ in June 2019 on indicators and reporting for surgery, obstetrics, and anesthesia patient safety. Although most of the previous Utstein papers focused on measuring performance (including nomenclature and definitions of important variables), the vision of an Utstein meeting for ensuring patients have access to safe anesthesia and surgery is to bring the same rigour and consensual thinking to surgical care as was previously brought to cardiac arrest and cardiopulmonary resuscitation. Thus, the meeting will be central to developing the metrics to facilitate national, international, inter-, and intra-system comparisons. It will act as a driver to quality improvement, identify gaps in knowledge, and support clinical research.

Ultimately, the intent is to ensure that the right data are being collected at country level to allow proper, informed, decision-making and a global, measurable, improvement in access to safe, affordable, timely anesthesia and surgical care.

\section{Reaching the five out of 7 billion people}

In 1980, then Secretary General of the WHO, Dr. Halfdan Mahler, addressed the World Congress of the International College of Surgeons and called the lack of access to surgical care the "most serious manifestation of social inequity in healthcare" ${ }^{17}$ Sadly, his reflection remains as true today as it was almost 40 years ago. 
Despite significant progress, many of the countries that are home to the $70 \%$ ( 5 billion) of the global population currently without access to safe anesthesia and surgery are the same countries that face the greatest difficulties with collecting and analysing data. Studies such as the Africa Surgical Outcomes Study ${ }^{18}$ point the way forward. Yet, much more can and should be done to ensure that LMIC governments, academic institutions, hospitals, and healthcare providers have the capacity to collect the right data, to ensure its comparability, and to enable analysis, sharing, and publication.

Juran et al. shine a valuable light on why data are so important and what might be done next. We join them in highlighting that the shocking inequity in global anesthesia and surgical provision will remain with us for as long as we are without the data to drive change.

\section{Les données comptent : implications pour la chirurgie et l'anesthésie pour atteindre la santé universelle}

Les données mesurent non seulement les changements, elles les inspirent et les ciblent. Sans données il est très difficile, sinon impossible, d'accélérer et de maintenir un progrès durable.

L'ère des Objectifs du millénaire pour le développement (OMD) a contribué à souligner l'importance des données pour le développement. ${ }^{1}$ Historiquement, la plupart des données collectées dans le monde incluent des mesures telles que la population, le produit national brut ou le produit intérieur brut par habitant qui sont principalement des indicateurs économiques ou démographiques. Ce n'est qu'ultérieurement que ces indicateurs ont commencé à inclure davantage de détails sur l'expérience humaine. L'un des changements les plus fondamentaux, et certainement l'un des plus influents, a eu lieu en 1990 avec la publication du premier Rapport mondial sur le développement humain ${ }^{2}$ et de l'Indice du développement humain (IDH). L'IDH (basé sur l'espérance de vie, le revenu par habitant et l'éducation) reste — presque 30 ans plus tard - une source importante de données et de comparaison entre les différents pays. Cela est dû, en grande partie au fait qu'il produit un classement composite basé sur seulement quelques indicateurs largement collectés, comparables et significatifs.

Les OMD ont été annoncés en septembre 2000 par l'Assemblée générale des Nations Unies. Le premier dispositif de suivi a d'abord inclus 18 cibles et
48 indicateurs couvrant huit objectifs globaux. Vingt-trois de ces indicateurs avaient un rapport avec la santé. L'objectif $\mathrm{n}^{\circ} 4$ était de « réduire la mortalité infantile » et l'objectif $\mathrm{n}^{\circ} 5$ était «d'améliorer la santé maternelle ». Les indicateurs apparentés étaient la «mortalité des enfants de moins de 5 ans » et le «taux de mortalité maternelle ». Ces indicateurs sont maintenant colligés par presque tous les pays du monde. Tous deux sont les principaux moteurs et outils de mesure du changement, avec des répercussions majeures sur les prises de décision pour la santé dans le monde et le financement d'objectifs de développement durable ratifiés en 2015 avec 232 indicateurs dont plus de 50 sont liés à la santé. ${ }^{3}$ Néanmoins, il n'y en a pas un seul parmi ces 50 indicateurs qui ait trait spécifiquement à l'anesthésie et à la chirurgie, alors qu'environ $33 \%$ de tous les décès enregistrés en 2010 étaient dus à des pathologies qui auraient pu être résolues par une intervention chirurgicale. ${ }^{4}$ Dans un autre ensemble d'indicateurs internationalement reconnus, la « liste mondiale de référence des 100 indicateurs sanitaires de base » de l'Organisation mondiale de la santé (OMS), ${ }^{5}$ seulement quatre font directement référence à l'anesthésie et à la chirurgie :

- Le taux de mortalité périopératoire : le taux de décès toutes causes confondues avant le congé parmi les patients ayant reçu au moins une procédure en salle d'opération pendant l'hospitalisation de référence;

- Le volume chirurgical : le nombre d'interventions et procédures pratiquées dans une salle d'opération par 100000 habitants et par an;

- L'accès à la chirurgie d'urgence : le pourcentage de la population ayant accès, dans un délai de deux heures, à un établissement pouvant réaliser en urgence un accouchement par césarienne, une laparotomie et la réparation d'une fracture ouverte;

- La densité des professionnels de santé et leur répartition : densité des professionnels de santé (c'està-dire, chirurgiens, anesthésiologistes et obstétriciens) par 10000 habitants.

Deux indicateurs supplémentaires (le pourcentage de la population confronté à des dépenses de santé catastrophiques ou entraînant leur appauvrissement) peuvent être analysés plus finement pour examiner les seules dépenses chirurgicales. Ces indicateurs sont également inclus dans les «Indicateurs du développement dans le monde » de la Banque mondiale. ${ }^{6}$

L'inclusion de ces quatre indicateurs a requis un effort concerté et nécessitera la poursuite d'un travail considérable pour leur collecte et analyse. C'est dans ce contexte que l'article de Juran et coll. publié dans ce numéro du Journal est à la fois bienvenu et opportun. ${ }^{7}$ Comme le font remarquer les auteurs, il semble que le 
monde a finalement reconnu la relation entre l'objectif d'une couverture de santé universelle d'ici 2030 et l'amélioration du système de santé. ${ }^{8}$ Néanmoins, il existe encore un hiatus entre l'atteinte d'un système de santé renforcé et la couverture dont nous voulons tous, avec la reconnaissance que cela doit inclure l'anesthésie et la chirurgie. L'une des raisons fréquemment citées pour ne pas le faire est son coût. Néanmoins, le coût estimé de 350 milliards de dollars pour une mise à niveau dans les pays à revenu intermédiaire et faible (PRIF) qui permettrait d'atteindre la cible de 5000 procédures par 100000 habitants et par an ne représente qu'une fraction des 12300 milliards de dollars d'économies qui pourraient être faites dans ces mêmes pays en évitant les pertes de productivité économique (perte cumulée estimée de 2015 à 2030). ${ }^{4}$ Cela ne représente également que $4 \%$ des 7830 milliards de dollars dépensés pour la santé en 2013. ${ }^{9}$ Avec les dépenses totales de santé qui devraient passer à 18300 milliards de dollars d'ici 2040, nous avons besoin de meilleures données pour nous assurer qu'une partie de cette augmentation ira à l'anesthésie et à la chirurgie dans le monde.

\section{La réponse}

Donc, le principal fossé des connaissances pour ce qui concerne les injustices chirurgicales soulignées par Juran et coll. est préoccupant. La Fédération mondiale des sociétés d'anesthésiologie (FMSA) est l'un des organismes qui ont travaillé à combler ce fossé. En septembre 2017, nous avons effectivement publié une enquête mondiale sur les effectifs en anesthésie (Global Anaesthesia Workforce Survey) qui a été la première tentative à vraiment rassembler des données au niveau mondial sur les prestataires d'anesthésie, tant médecins que non-médecins. ${ }^{10}$ Cela s'accompagnait d'une carte en ligne des effectifs ${ }^{11}$ qui offre une présentation visuelle interactive et actualisée des endroits du monde où le manque d'anesthésiologistes est le plus criant. De cette façon, l'enquête et la carte deviennent des outils utiles pour influencer des changements et mesurer leurs progrès. La FMSA a l'intention d'actualiser régulièrement la carte et de répéter l'enquête tous les quatre ans. La répétition de l'enquête assure également que nous pouvons l'adapter et l'améliorer. Nous espérons, par exemple, collecter les mêmes données sur les personnels, mais en les analysant selon le sexe lorsque nous reprendrons l'enquête en 20192020.

De plus, la FMSA a créé un outil d'évaluation des établissements d'anesthésie ou AFAT (Anaesthesia Facility Assessment Tool). ${ }^{12}$ S'appuyant sur les normes internationales 2018 pour une pratique sécuritaire de l'anesthésie de l'OMS-WFSA/FMSA, ${ }^{13}$ récemment mises à jour, la FMSA a élaboré l'AFAT pour aider l'anesthésie en région et au niveau national, ainsi que le leadership dans le domaine de la santé, à réunir des données complètes sur les effectifs, les équipements, les médicaments et les pratiques en anesthésie au niveau des établissements. L'AFAT fait partie d'un effort commun pour améliorer la collecte des données et la gestion des connaissances visant à soutenir la mise en œuvre de la Résolution 68.15 de l'Assemblée mondiale de la santé qui appelle tous les états membres à renforcer les soins chirurgicaux et anesthésiques essentiels et urgents dans le cadre de la couverture de santé universelle. ${ }^{14}$ Elle contribuera également à assurer que l'anesthésie est représentée dans les programmes sanitaires nationaux incluant des plans nationaux pour la chirurgie, l'obstétrique et l'anesthésie. ${ }^{15}$

\section{Une action collective pour la collecte de données}

Tout ceci repose sur un partenariat et un travail en équipe, non seulement des équipes chirurgicales, mais aussi des gouvernements et organisations internationales, du monde académique, des organisations non gouvernementales (ONG), des patients, etc. L'OMS, la Commission des statistiques des Nations Unies et la Banque mondiale sont les pivots de cet effort. Ils devront travailler ensemble pour s'assurer que non seulement les indicateurs anesthésiques et chirurgicaux soient inclus dans leurs ensembles de données, mais aussi que nous collections, partagions et analysions les mêmes données disponibles, comparables et utiles dans l'ensemble du système de santé mondial.

Juran et coll. insistent sur la nécessité d'un séminaire ou d'une conférence internationale. À ce sujet, la FMSA travaille en étroite collaboration avec la Fondation Laerdal, l'OMS, le King's College de Londres, les anciens dirigeants de la Lancet Commission on Global Surgery, les universités de Lund et de Gothenburg, la Harvard Medical School et l'équipe d'anesthésie internationale de l'université de Californie à San Francisco, et d'autres encore, pour convoquer une réunion à Utstein ${ }^{16}$ en juin 2019 sur les indicateurs et les rapports pour la sécurité des patients en chirurgie, obstétrique et anesthésie. Même si la majorité des précédents articles «Utstein » se concentraient sur la mesure des performances (y compris la nomenclature et la définition des variables importantes), l'espoir d'une rencontre à Utstein pour garantir que les patients ont accès à une chirurgie et une anesthésie sécuritaires est d'apporter la même rigueur et la même réflexion consensuelle aux soins chirurgicaux que celles qui ont été accordées antérieurement à l'arrêt cardiaque et à la ressuscitation cardiopulmonaire. Ainsi, cette réunion jouera un rôle central dans l'élaboration des paramètres de mesure 
facilitant des comparaisons entre et à l'intérieur des systèmes, aux niveaux nationaux et internationaux. Cela sera un moteur de l'amélioration de la qualité, identifiera les lacunes dans les connaissances et soutiendra la recherche clinique.

Enfin, le but est de s'assurer que les bonnes données soient collectées au niveau de chaque pays pour permettre des prises de décisions adéquates et informées, ainsi qu'une amélioration globale, mesurable de l'accès abordable et opportun à des soins anesthésiques et chirurgicaux sécuritaires.

\section{Atteindre 5 milliards de personnes (sur 7)}

En 1980, le secrétaire général de l'OMS, le docteur Halfdan Mahler, s'est adressé au Congrès mondial du Collège international de chirurgie et a appelé l'absence d'accès aux soins chirurgicaux la "manifestation la plus grave de l'inégalité sociale dans les soins de santé $»{ }^{17}$ Malheureusement, sa réflexion reste d'actualité presque 40 ans plus tard.

En dépit de progrès significatifs, de nombreux pays abritant $70 \%$ de la population mondiale, soit 5 milliards d'habitants sans accès à des soins anesthésiques et chirurgicaux sécuritaires - sont ces mêmes pays qui sont confrontés aux plus grandes difficultés de collecte et d'analyse des données. Des études telles que l'Africa Surgical Outcomes Study ${ }^{18}$ montrent la voie à suivre. Il est cependant possible de faire beaucoup plus et on doit le faire pour s'assurer que les gouvernements des PRIF, les établissements universitaires, les hôpitaux et les prestataires de soins de santé auront la capacité de collecter les bonnes données permettant leurs comparaisons, leur analyse, communication et publication.

Juran et coll. éclairent de façon intéressante pourquoi les données sont si importantes et ce que l'on pourrait faire ensuite. Nous les rejoignons pour souligner que les inégalités choquantes qui existent dans la fourniture de soins anesthésiques et chirurgicaux dans le monde persisteront tant que nous ne disposerons pas des données pour provoquer le changement.

Conflicts of interest None declared.

Editorial responsibility This submission was handled by Dr. Hilary P. Grocott, Editor-in-Chief, Canadian Journal of Anesthesia.

Conflits d'intérêts Aucun déclaré.

Responsabilité éditoriale Cet article a été traité par le Dr Hilary P. Grocott, Rédacteur en chef, Journal canadien d'anesthésie.

\section{References}

1. United Nations. United Nations Millennium Declaration (September 2000). Available from URL: http://www.un.org/ millennium/declaration/ares552e.pdf (accessed September 2018).

2. Kaul I, Goldstone L, Hausner B, et al. Human Development Report, 1990. United Nations Development Programme (UNDP). Oxford University Press. Available from URL: http://hdr.undp. org/sites/default/files/reports/219/hdr_1990_en_complete_nostats. pdf (accessed September 2018).

3. Sustainable Development Goals. SDG Indicators. Global indicator framework for the Sustainable Development Goals and targets of the 2030 Agenda for Sustainable Development. Available from URL: https://unstats.un.org/sdgs/indicators/ indicators-list/ (accessed September 2018).

4. Meara JG, Leather AJ, Hagander L, et al. Global surgery 2030: evidence and solutions for achieving health, welfare and economic development. Lancet 2015; 386: 569-624.

5. World Health Organization. Global Reference List of 100 Core Health Indicators, 2015. Available from URL: http://www.who. int/healthinfo/indicators/2015/en/ (accessed September 2018).

6. The World Bank. World Development Indicators. Available from URL: https://data.worldbank.org/products/wdi (accessed September 2018).

7. Juran S, Gruendl M, Marks I, et al. The need to collect, aggregate, and analyze global anesthesia and surgery data. Can J Anesth 2018; 65. DOI https://doi.org/10.1007/s12630-018-12615.

8. World Health Organization. Universal health coverage. Key facts. Available from URL: http://www.who.int/news-room/factsheets/detail/universal-health-coverage-(uhc) (accessed September 2018).

9. Dieleman JL, Templin T, Sadat N, et al. National spending on health by source for 184 countries between 2013 and 2040 . Lancet 2016; 387: 2521-35.

10. Kempthorne P, Morriss WW, Mellin-Olsen J, Gore-Booth J. The WFSA Global Anesthesia Workforce Survey. Anesth Analg 2017; 125: 981-90.

11. World Federation of Societies of Anaesthesiologists. World Anaesthesiology Workforce Map. Available from URL: https:// www.wfsahq.org/workforce-map (accessed September 2018).

12. World Federation of Societies of Anaesthesiologists. Anaesthesia Facility Assessment Tool. Available from URL: https://www. wfsahq.org/afat (accessed September 2018).

13. Gelb AW, Morriss WW, Johnson W, Merry AF, International Standards for a Safe Practice of Anesthesia Workgroup. World Health Organization-World Federation of Societies of Anaesthesiologists (WHO-WFSA) International Standards for a Safe Practice of Anesthesia. Can J Anesth 2018; 65: 698-708.

14. WHA Resolution 68.15. Strengthening Emergency and Essential Surgical Care and Anaesthesia as a Component of Universal Health Coverage. Geneva, Switzerland: World Health Assembly; 2015. Available from URL: http://apps.who.int/gb/ebwha/pdf files/wha68/a68_r15-en.pdf (accessed September 2018).

15. World Health Organization. Surgical care systems strengthening: developing national surgical, obstetric and anaesthesia plans. World Health Organization - 2017. Available from URL: http:// www.who.int/iris/handle/10665/255566 (accessed September 2018). License: CC BY-NC-SA 3.0 IGO.

16. Dick WF, Baskett PJ, Grande C, et al. Recommendations for uniform reporting of data following major trauma-the Utstein style" (as of July 17, 1999). An International Trauma Anaesthesia and Critical Care Society (ITACCS). Acta Anaesthesiol Belg 2000; 2000(51): 18-38 
17. World Health Organization. Address by Dr H Mahler to the XXII Biennial World Congress of the International College of Surgeons. Available from URL: http://www.who.int/surgery/ strategies/Mahler1980speech.pdf (accessed September 2018).
18. Biccard BM, Madiba TE, Kluyts $H G$, et al. Perioperative patient outcomes in the African Surgical Outcomes Study: a 7-day prospective observational cohort study. Lancet 2018; 391: 158998. 